\title{
Farklı Optimizasyon Teknikleri ve Örneklem Büyüklüklerinde Standart Tobit Modeli Tahmincilerinin Performansı üzerine Bir Simülasyon Çalışması
}

\author{
On the Performance of Estimators in Standard Tobit Model under \\ Different Optimization Techniques and Sample Sizes: A Simulation \\ Study
}

\author{
Büşra Emir, Ertuğrul Çolak, Kevser Setenay Öner
}

Eskişehir Osmangazi Üniversitesi Tıp Fakültesi Biyoistatistik Anabilim Dalı, Eskişehir, Türkiye

Özet: Bu çalışmanın amacı, bağımlı değişkenin sınırlı bir değer aralığında gözlemlendiği regresyon modelinde kullanılan parametre tahmin yöntemlerini karşılaştırmaktır. Bu model Tobit model ya da sansürlü regresyon modeli olarak adlandırılmaktadır. Tobit modelde rasgele hatanın 0 ortalama ve $\sigma^{2}$ varyans ile normal dağılım gösterdiği varsayılmaktadır. Bu varsayıma göre simülasyon çalışması yapılmıştır. Simülasyon çalışmasının amacı, hangi yöntemin parametreleri tahmin etmede en iyi olduğunu belirlemektir. Kullanılan parametre tahmin yöntemleri; Probit en büyük olabilirlik yöntemi, Tobit en büyük olabilirlik yöntemi ve Heckman iki aşamalı tahmin yöntemidir. Olabilirlik fonksiyonları, parametrelerin doğrusal bir fonksiyonu olmadıkları için bu modellerin açık çözümleri elde edilememektedir. Bu nedenle, bu tür modellerin parametre tahminleri için farklı optimizasyon teknikleri geliștirilmiş̦tir. Bu çalışmada, Newton Raphson (NR), Quasi Newton (QN), Conjugate Gradient (CG), Double Dogleg (DD), Nelder Mead Simplex (NM), Newton Raphson Ridging (NRR) ve Trust Region (TR) optimizasyon teknikleri kullanılmıştır. Karşılaștırmalar iki temel ölçüt kullanılarak yapılmıștır. Bu ölçütler, parametre tahminleri ve hata kareler ortalamaları, yakınsama oranlarına göre optimizasyon tekniklerinin performansları olarak belirlenmiştir. Tobit en büyük olabilirlik yöntemi için örneklem büyüklüğ̈̈ 50 iken, optimizasyon tekniklerinin tümü ile başlangıç parametre değerlerine oldukça yakın ortalama değerlerine ulaşılmıştır. Hata kareler ortalama değerleri de diğer yöntemlere göre oldukça küçük bulunmuş̧ur. Tobit en büyük olabilirlik yöntemi, diğer tahmin yöntemine göre örneklem büyüklüğ̈̈ çok küçük olduğunda bile parametreleri tahmin etmede en iyi yöntemdir. Optimizasyon tekniklerinin yakınsama oranları incelendiğinde, en iyi performans gösteren optimizasyon tekniği Quasi Newton'dur.

Anahtar Kelimeler: parametre tahmin yöntemleri, probit en büyük olabilirlik yöntemi, tobit en büyük olabilirlik yöntemi, heckman iki aşamalı tahmin yöntemi, optimizasyon teknikleri

Emir B, Çolak E, Öner K S. 2018, Farklı Optimizasyon Teknikleri ve Örneklem Büyüklüklerinde Standart Tobit Modeli Tahmincilerinin Performansı üzerine Bir Simülasyon Çalışması, Osmangazi Tip Dergisi, 40 (2):60-69, Doi: 10.20515/otd.409064

Abstract: The purpose of this study is to compare the parameter estimation methods used in the regression model where the dependent variable is observed in a limited range of values. This model is called Tobit model or censored regression model. It is assumed that the random error has a normal distribution with 0 mean and $\sigma^{2}$ variance in the Tobit model. According to this assumption, simulation study has been done. The aim of the simulation study is to determine which method is best to estimate the parameters. These estimation methods included Probit Maximum Likelihood, Tobit Maximum Likelihood and Heckman's TwoStep. It can not be obtained clear solutions of these models since the likelihood functions are not a linear function of the parameters. For this reason, different optimization techniques have been developed for parameter estimation of such models. In this study, it is used Newton Raphson (NR), Quasi Newton (QN), Conjugate Gradient (CG), Double Dogleg (DD), Nelder Mead Simplex (NM), Newton Raphson Ridging (NRR) and Trust Region (TR) optimization techniques. Comparisons were made using two basic criteria. These criteria were determined as parameter estimates and mean square error, performances of optimization techniques according to convergence rates. In the Tobit maximum likelihood estimation method, all of the optimization techniques have reached mean values very close to the initial parameter values when the sample size has 50 . The values of mean square error were found to be quite small compared to other methods.Tobit maximum likelihood is the best method to estimate parameters even when the sample size is very small compared to other estimation methods. The best performing optimization technique is Quasi Newton when the convergence rates of the optimization techniques are examined.

Keywords: parameter estimation methods, probit maximum likelihood estimation method, tobit maximum likelihood estimation method, heckman two step estimation method, optimization techniques

Emir B, Colak E, Oner K S. 2018, On the Performance of Estimators in Standard Tobit Model under Different Optimization Techniques and Sample Sizes: A Simulation Study, Osmangazi Journal of Medicine, 40 (2):60-69， Doi: 10.20515/otd.409064

60

Geliş Tarihi / Received 23.03.2018
Kabul Tarihi / Accepted: 12.04.2018
Yazışma Adresi / Correspondence Address

Büşra EMİR

mail: bemir@ogu.edu.tr 


\section{Giriş}

Regresyon modelleri, uygulamalarda siklıkla karşılaştığımız en çok kullanılan istatistiksel modellerden biridir. Modellerdeki bağımlı ve bağımsız değişkenlerin sayısına ve türüne bağlı olarak çok çeşitli regresyon modelleri geliştirilmiştir. Bunlardan biri tobit modelidir. $\mathrm{Bu}$ model 1958 yılında Nobel ödüllü iktisatçı James Tobin tarafından geliştirilmiştir ve bağımlı değişken değerlerinin elde edilmesi açısından diğer regresyon modellerinden farklıdır (1). Model son yıllarda uygulama alanına girmiştir. Tobin'in Probit modellerinin bir uzantısı olarak geliştirdiği bu yöntem, 1964 y1lında Goldberger tarafindan Tobin ve Probit isimlerinden türetilmiştir (2). Tobit modeli, doğrusal regresyon modellerinin aksine bağımsız değişkenin bilinen değerlerine uygun olarak bağımlı değişken değerlerinin bazılarının gözlemlenemediği durumlarda kullanılır. $\mathrm{Bu}$ modellerde sürekli bağımlı değişken değerleri bazen üstten bazen alttan sansürlüdür. Fakat bağımsız değişkenlerin elde edilmesinde bir sinırlama bulunmamaktadır. Veri sansürü meydana geldiğinde, en küçük kareler doğrusal regresyon modelleri tahmincilerinin yanlı ve tutarsız olduğu birçok araştırmada simülasyon çalışmaları ile kanıtlanmıştır (3-5).

Uluslararası alanlarda yapılan çalışmalarda, genellikle EQ-5D (EuroQol five-dimension scale), EQ-VAS (Visual Analogic Scale) gibi yaşam kalitesini gösteren ölçütlerin tahmin edilmesinde ve bağımlı değişkenin sansürlü bir yapıya sahip olduğu durumlarda tobit modeli kullanılmıştır (6-8). Ancak ülkemizde sağlık alanında yapılan araştırmalarda tobit modellerinin kullanımının sinırlı olduğu gözlemlenmiştir. Sağlık alanında yapılan araştırmalarda tahmin edilecek bağımlı değişken değerlerinin alttan ve üstten sansürlenmesi durumları ile çok sık karşılaşılmaktadır. $\mathrm{Bu}$ yapıyı göz önüne almadan farklı yöntemler ile analizler gerçekleştirildiğinde yanlış bulgular ve sonuçlar ortaya çıkmaktadır. Tobit modelinin sağlık alanında kullanımını artırarak doğru sonuçlar elde edilebilmesi mümkündür.

Amemiya, bağımlı değişkenin sansürlenme yapısına göre beş farklı tobit modeli tanımlamıştır (9). Bu çalışmada Tip-1 tobit modeli olarak adlandırılan standart tobit modeli incelenmiştir.

Parametre tahmin yöntemleri standart tobit modeli esas alınarak gerçekleştirilmiştir. Standart tobit modeli, $y_{i}{ }^{*}$ gibi bir gizli (latent) değişkenin varlığını varsayar $(i=1,2, \ldots, n)$. $\mathrm{Bu}$ değişken $\boldsymbol{x}_{\boldsymbol{i}}$ bağımsı $\mathrm{K}$ değişkenine doğrusal olarak $\boldsymbol{\beta}$ parametreler vektörü ile bağlıdır $\left(x_{i}{ }^{\prime}=\left(1, x_{i}{ }^{1}, \ldots, x_{i}{ }^{K}\right), \boldsymbol{\beta}=\left(\beta_{0}, \beta_{1}, \ldots, \beta_{K}\right)^{\prime} \in \Re^{K}\right)$. $\boldsymbol{\beta}$ vektörü doğrusal modelde olduğu gibi $x_{i}$ ve $y_{i}$ arasındaki ilişkiyi belirler. Ayrıca bu ilişkideki rassal etkileri kapsayacak normal dağılıma sahip bir hata terimi $\varepsilon_{\mathrm{i}}$ vardır $\left(\varepsilon_{i} \mid x_{i} \sim \mathrm{N}\left(0, \sigma^{2}\right)\right.$. Standart tobit modelinin matematiksel formu:

$$
y_{i}= \begin{cases}y_{i}{ }^{*} & , y_{i}^{*}>0 \text { ise } \\ 0 & , y_{i}{ }^{*} \leq 0 \text { ise }\end{cases}
$$

Burada,

$$
y_{i}^{*}=\boldsymbol{x}_{\boldsymbol{i}}^{\prime} \boldsymbol{\beta}+\varepsilon_{i}
$$

Standart tobit modelinde parametre tahminleri olabilirlik yöntemleri kullanılarak elde edilir. $\mathrm{Bu}$ yaklaşımın genel geçerliliği 2005 yılında Schnedler tarafından gösterilmiştir (10). Olabilirlik fonksiyonları parametrelerin doğrusal bir fonksiyonu olmadığından bu modellerin açık çözümleri elde edilemez. Bu nedenle, bu tür modellerin parametre tahminleri için farklı optimizasyon teknikleri geliştirilmiştir (11). Bu bilgiler doğrultusunda, farklı optimizasyon teknikleri ve örneklem büyüklükleri altında standart tobit modelinde parametre tahmin yöntemlerinin performanslarının araştırılması amaçlanmıştır.

\section{Gereç ve Yöntemler}

$\mathrm{Bu}$ bölüm, standart tobit modelinde parametre tahmini için kullanılan Probit en büyük olabilirlik yöntemi, Tobit en büyük olabilirlik yöntemi, Heckman iki aşamalı tahmin yöntemi, Optimizasyon teknikleri ve Simülasyon çalışmasından oluşmaktadır.

\subsection{Probit En Büyük Olabilirlik Yöntemi}

Tobit ve Probit modelleri birçok yönden benzerlik gösterir. Hata terimleri $\varepsilon$ her iki modelde de standart normal dağııma sahip olduğu varsayılmaktadır. Ayrıca, $y_{i}{ }^{*}$ gibi bir gizli (latent) değişken kullanmaktadır. 
$y_{i}{ }^{*}$ değişkeninin nasıl $y_{i}$ değişkenine dönüştürüleceği farkl1lık göstermektedir.

$$
y_{i}= \begin{cases}1, & y_{i}{ }^{*}>0 \text { ise } \\ 0, & y_{i}{ }^{*} \leq 0 \text { ise }\end{cases}
$$

Tobit modelde $y_{i}{ }^{*}>0$ olduğunda $y_{i}{ }^{*}$ değeri bilinirken, Probit modelde sadece $y_{i}{ }^{*}$ gizli (latent) değişkeninin 0 'dan büyük olup olmadığı bilinmektedir, $y_{i}{ }^{*}$ değeri bilinmemektedir.

$\mathrm{F}\left(\boldsymbol{x}_{\boldsymbol{i}}^{\prime} \boldsymbol{\beta}\right)$, birikimli dağılım fonksiyonunu ifade etsin. Modeldeki bilinmeyen $\boldsymbol{\beta}$ parametrelerini tahmin etmek için Probit modelinin olabilirlik ve logaritmik olabilirlik fonksiyonları sırasıyla aşağıdaki gibi elde edilir $(12,13)$.

$$
\begin{aligned}
\ell(\boldsymbol{\beta})= & \prod_{i=1}^{\mathrm{N}} \mathrm{F}\left(\boldsymbol{x}_{\boldsymbol{i}}{ }^{\prime} \boldsymbol{\beta}\right)^{y_{i}}\left[1-\mathrm{F}\left(\boldsymbol{x}_{\boldsymbol{i}}{ }^{\prime} \boldsymbol{\beta}\right)\right]^{1-y_{i}} \\
\mathcal{L}(\boldsymbol{\beta})= & \ln [\ell(\boldsymbol{\beta})]=\sum_{i=1}^{N} y_{i} \ln \mathrm{F}\left(\boldsymbol{x}_{\boldsymbol{i}}{ }^{\prime} \boldsymbol{\beta}\right) \\
& +\left(1-y_{i}\right) \ln \left(1-\mathrm{F}\left(\boldsymbol{x}_{\boldsymbol{i}}{ }^{\prime} \boldsymbol{\beta}\right)\right)
\end{aligned}
$$

Parametre tahminleri, log-olabilirlik fonksiyon-una göre türevi sıfir yapan nokta ile elde edilmektedir.

\subsection{Tobit En Büyük Olabilirlik Yöntemi}

Probit modelinde, hata terimlerinin normal dağılıma sahip olduğu varsayıldığında, $\frac{\beta_{0}}{\sigma}$ ve $\frac{\beta_{K}}{\sigma}$ için tutarlı ve asimptotik normal dağılımlı tahminler elde etmek mümkündür $\left(\varepsilon_{i} \sim \mathrm{N}\left(0, \sigma^{2}\right)\right)$. Ancak, $\quad \beta \quad$ regresyon parametrelerini ve $\sigma$ standart sapmayı ayrı bir şekilde tahmin etmek mümkün değildir.

Amemiya (1973), tobit modelin log-olabilirlik fonksiyonunun $\beta$ ve $\sigma$ parametrelerine göre yakınsama sağlamadığını (globally concave) göstermiş̧ir. Olsen'in tekrar parametre dönüştürmesinden sonra, tobit model, NewtonRaphson gibi standart iteratif optimizasyon teknikleri ile her zaman logaritmik olabilirlik fonksiyonunun global maksimum değerine yakınsadığını göstermiştir $(14,15)$. Tobit modelin olabilirlik ve logaritmik olabilirlik fonksiyonları sırasıyla aşağıdaki gibi elde edilir (16).

$$
\begin{aligned}
& \ell(\boldsymbol{\beta})= \prod_{i=1}^{\mathrm{N}}\left\{\frac{1}{\sigma} \varphi\left(\frac{y_{i}-\boldsymbol{x}_{i}^{\prime} \boldsymbol{\beta}}{\sigma}\right)\right\}^{d_{i}} \cdot\left\{1-\Phi\left(\frac{\boldsymbol{x}_{\boldsymbol{i}}^{\prime} \boldsymbol{\beta}}{\sigma}\right)\right\}^{1-d_{i}} \\
& \mathcal{L}(\boldsymbol{\beta})=\ln [\ell(\boldsymbol{\beta})]=\sum_{i=1}^{\mathrm{N}}\left[d_{i} \ln \left\{\frac{1}{\sigma} \varphi\left(\frac{y_{i}-\boldsymbol{x}_{\boldsymbol{i}}^{\prime} \boldsymbol{\beta}}{\sigma}\right)\right\}\right. \\
&\left.+\left(1-d_{i}\right) \ln \left\{1-\Phi\left(\frac{\boldsymbol{x}_{\boldsymbol{i}}^{\prime} \boldsymbol{\beta}}{\sigma}\right)\right\}\right]
\end{aligned}
$$

Burada $\varphi, \Phi$ ve $d_{i}$ sırası ile olasılık dağılım fonksiyonunu, birikimli dağılım fonksiyonunu ve $y_{i}>0$ olduğunda 1 , diğer durumlarda 0 olan yapay (dummy) değişkeni ifade etmektedir.

\subsection{Heckman İki Aşamalı Tahmin Yöntemi}

Heckman iki aşamalı tahmin yöntemi, Tobit en büyük olabilirlik yöntemine alternatif bir yöntemdir. Örneklem seçimindeki yanlılığın düzeltilmesi için kullanılan bir tahmin yöntemidir. Tahmin süreci iki aşamadan oluşmaktadır.

1.Aşama: Örneklem seçim sapmasını belirlemek ve seçim sapmasını gösteren modelin istatistiksel olarak doğruluğunu tespit etmek amacı ile seçim modeli tahmin edilir. Tüm veriler kullanılarak bir Probit modeli $\left(d_{i}=x_{i}^{\prime} \beta+\varepsilon_{i}\right)$ oluşturulur ve Probit en büyük olabilirlik yöntemi ile parametre tahminleri elde edilir.

2.Aşama: Bir düzeltme faktörü olan hazard fonksiyonu ya da Ters Mill's Oranı (Inverse Mill's Ratio) $\lambda$, Probit modelinden elde edilen sonuçlar kullanılarak bulunur ve ikinci adımda gözlemlerin yanlı tahminini ortadan kaldırmak amaciyla tahmin edilen hazard fonksiyonu $\lambda$, ayrı bir bağımsız değişken olarak modele dahil edilir. $y_{i}>0$ olan değerler için ikinci bir model oluşturulur.

$$
y_{i}=\boldsymbol{x}_{\boldsymbol{i}}{ }^{\prime} \boldsymbol{\beta}+\sigma \cdot \lambda\left(\frac{\widehat{\boldsymbol{x}_{\boldsymbol{i}} \boldsymbol{\beta} \boldsymbol{\beta}}}{\sigma}\right)+\varepsilon_{i}
$$

Model oluşturulduktan sonra en küçük kareler yöntemi ile parametre tahminleri elde edilir (17).

\subsection{Optimizasyon Teknikleri}

Doğrusal regresyon analizinde bilinmeyen parametreler için elde edilen olabilirlik fonksiyonları doğrusaldır ve çözümleri 
kolaydır. Ancak bağımlı değişkeni sansürlü, sınırlandırılmış ya da kategorik yapıda olan modellerin olabilirlik fonksiyonları parametrelerin doğrusal fonksiyonları olmadıkları için açık çözümleri elde edilemez. Bu nedenle bu tür modellerin parametre tahminlerine ilişkin Newton Raphson (NR), Quasi Newton (QN), Conjugate Gradient (CG), Double Dogleg (DD), Nelder Mead Simplex (NM), Newton Raphson Ridging (NRR) ve Trust Region (TR) optimizasyon teknikleri gibi birçok optimizasyon teknikleri geliştirilmiştir.

Doğrusal olmayan eşitlik çözümlerinde kullanılan optimizasyon teknikleri, iterasyon boyunca kullandıkları bilgilere göre farklılık göstermektedir. Genel olarak, optimizasyon teknikleri farkl1 Gradient ve Hessian matrisine ait bilgileri kullanarak yakınsama sağlarlar. Parametre tahmininde öncelikle parametreler için başlangıç değerleri atanır. Her iterasyonda atanan bu parametre değerleri güncellenir. Amaç hata olabilirlik fonksiyonunu maksimize etmek yani hata fonksiyonunu minimize etmektir. Örneğin Trust Region tekniği hata fonksiyonunun parametre tahminlerine göre birinci ve ikinci derecede türevleri için bir bölge tanımlar ve parametrelerin tahmin edilmesini sağlar. Newton Raphson Ridge tekniği Hessian matrisinin ortogonal parçalanmasından yararlanmaktadır. DoubleDogleg tekniği, Quasi Newton ile TrustRegion yöntemlerini bir araya getirerek hata fonksiyonunu minimize etmektedir. Quasi Newton, Conjugate Gradient ve Double Dogleg birinci dereceden türev gerektiren tekniklerdir. Newton Raphson, Newton Raphson Ridging, Trust Region teknikleri birinci ve ikinci dereceden türevlenebilir yapida olan hata fonksiyonlarına uygulanmaktadır. Nelder Mead Simplex tekniği ise hata fonksiyonunun sürekli türevlenebilir olmasını varsaymamaktadır. $\mathrm{Bu}$ nedenle birinci ve ikinci dereceden türev gerektirmez (11).

\subsection{Simülasyon Çalışması}

Simülasyon çalışmasında kullanılmak üzere tek bağımsız değişken içeren standart tobit modeli aşağıdaki gibi oluşturulmuştur.

$$
y_{i}^{*}=\beta_{0}+\beta_{1} x_{i}+\varepsilon_{i}
$$

$$
y_{i}= \begin{cases}y_{i}{ }^{*} & , y_{i}{ }^{*}>0 \text { ise } \\ 0 & , y_{i}{ }^{*} \leq 0 \text { ise }\end{cases}
$$

Burada $\beta_{0}$ ve $\beta_{1}$, model parametrelerini, $\varepsilon_{i}$, modelde yer alan rassal değişkeni, 0 ortalamalı ve $\sigma^{2}$ varyanslı normal dağılıma sahip rasgele etkiyi göstermektedir ve $x_{i}$ açıklayıcı değişkeninden bağımsızdır $\left(\varepsilon_{i} \mid x_{i} \sim \mathrm{N}\left(0, \sigma^{2}\right)\right.$.

Parametre tahmin yöntemlerinin performanslarını incelemek için aşağıdaki simülasyon adımları uygulanmıştır.

\section{Simülasyon Adımları}

1. Başlangıç parametre değerleri $\beta_{0}=1$, $\beta_{1}=1$ ve $\sigma^{2}=1$ olarak belirlendi.

2. $n_{i}$ örneklem büyüklüğüne sahip $x_{i}$ bağımsız değişken ve $\varepsilon_{i}$ rassal değişkeni standart normal dağılımdan türetildi.

3. İlk iki adımda elde edilen değerler, $y_{i}{ }^{*}$ gizli (latent) değişkeni ve $y_{i}$ cevap değişkeni elde etmek için standart tobit modeli oluşturuldu.

4. Probit en büyük olabilirlik yöntemi, Tobit en büyük olabilirlik yöntemi ve Heckman iki aşamalı tahmin yöntemlerini içeren parametre tahmin yöntemleri ikinci adımda elde edilen açılayıcı değişken ve üçüncü adımda elde edilen cevap değişkeni kullanılarak analizler gerçekleştirildi.

5. İlk dört adım bağımsız olarak $1000 \mathrm{kez}$ tekrarlanmıştır. Buna göre, her bir yöntemden 1000 farklı parametre tahmini ve hata kareler ortalamaları elde edildi.

Böylece, bu 5 simülasyon adımı gerçekleştirilerek, parametre tahminlerinin ortalamalarının $\beta_{0}, \beta_{1}$ ve $\sigma^{2}$ başlangıç değerine ne kadar yakınsadığı araştırıldı. Örneklem büyüklükleri sirasılyla $n_{i}=50,100,250$ ve 500 birim olarak belirlendi. Simülasyon çalışması PROC QLIM prosedürü ile SAS 9.3 programı kullanılarak gerçekleştirildi. PROC QLIM prosedürü, doğrusal olmayan optimizasyon tekniklerini gerçekleştirmek için doğrusal olmayan optimizasyon (Nonlinear Optimization-NLO) alt sistemini kullanmaktadır. NLOPTIONS deyiminde NLO seçeneklerinden 7 farklı optimizasyon tekniği bulunmaktadır. Böylece, tüm optimizasyon 
teknikleri PROC QLIM'de uygulandı ve yakınsama oranlarına göre optimizasyon tekniklerinin performansları karşılaştırıldı.

\section{Bulgular}

Simülasyon çalışması sonucunda Probit en büyük olabilirlik, Tobit en büyük olabilirlik ve Heckman iki aşamalı tahmin yöntemleri için elde edilen parametre tahminlerinin deneysel ortalamaları ve hata kareler ortalamaları sırası ile Tablo 1 ve Tablo 2'de gösterilmiştir.

Probit en büyük olabilirlik yöntemi için parametre tahminlerinin deneysel ortalamaları ve hata kareler ortalamaları, örneklem büyüklüklerine göre sırası ile incelendiğinde; örneğin küçük bir veri seti varlığında, örneklem büyüklüğü 50 olduğunda, CG, DD ve QN optimizasyon teknikleri kullanıldığında başlangıç parametre değerlerine en yakın ortalama değerlerine ulaşılmıştır. NR, NM, NRR ve TR optimizasyon teknikleri kullanıldığında parametre tahminlerinin deneysel ortalamaları aynı sonuçları vermiştir. Örneklem büyüklüğü 100 incelendiğinde, NR, DD, NM, NRR ve TR optimizasyon teknikleri ile parametre tahmin değerleri aynı bulunmuştur. QN ve CG optimizasyon teknikleri kullanıldığında başlangıç parametre değerlerine en yakın ortalama değerlerine ulaşılmıştır. Örneklem büyüklüğü arttıkça, deneysel ortalamalar başlangıç parametre değerlerine oldukça yakınsamıştır ve hata kareler ortalama değerleri giderek azaldığ gözlemlenmiştir. NR, DD, NM, NRR ve TR optimizasyon teknikleri, her bir örneklem büyüklüğü kendi içerisinde incelendiğinde aynı parametre tahmin sonuçları elde ettikleri görülmüştür. Sirası ile QN ve CG optimizasyon teknikleri kullanılarak en yakın başlangıç parametre tahmin değerlerine ve en küçük hata kareler ortalama değerlerine ulaşı1mıştır.

Tobit en büyük olabilirlik yöntemi için parametre tahminlerinin deneysel ortalamaları ve hata kareler ortalamaları, örneklem büyüklüklerine göre sirası ile incelendiğinde; örneklem büyüklüğü 50 iken, optimizasyon tekniklerinin tümü ile başlangiç parametre değerlerine oldukça yakın ortalama değerlerine ulaşılmıştır. Hata kareler ortalama değerleri de diğer yöntemlere göre oldukça küçük bulunmuştur. Örneklem büyüklüğü arttıkça, deneysel ortalamalar başlangıç parametre değerlerine oldukça iyi yakınsamıştır ve hata kareler ortalama değerleri giderek azaldığ gözlemlenmiştir.

NM, NRR ve TR optimizasyon teknikleri, her bir örneklem büyüklüğü kendi içerisinde incelendiğinde aynı parametre tahmin sonuçları elde ettikleri görülmüştür. Sırası ile QN ve CG optimizasyon teknikleri kullanılarak en yakın başlangıç parametre tahmin değerlerine ve en küçük hata kareler ortalama değerlerine ulaşılmıştır.

Heckman iki aşamalı tahmin yöntemi için parametre tahminlerinin deneysel ortalamaları ve hata kareler ortalamaları, örneklem büyüklüklerine göre sırası ile incelendiğinde; örneklem büyüklüğü 50 iken, başlangıç parametre değerlerine en yakın ortalama değerlerine ve en düşük hata kareler ortalama değerlerine Trust Region (TR) optimizasyon tekniği kullanılarak ulaşılmıştır. Diğer optimizasyon teknikleri incelendiğinde ise, parametre tahmin değerleri yaklaşık olarak birbirine benzer sonuçlar vermiştir. Fakat diğer yöntemlerde olduğu gibi birebir aynı sonuçlar gözlemlenmemiştir. $\mathrm{Bu}$ durumun sebebi, örneklem seçim yanlılığını ortadan kaldırmak için kullanılan iki aşamadan oluşan tahmin sürecidir. Örneklem büyüklüğü arttıkça, deneysel ortalamalar başlangıç parametre değerlerine oldukça iyi yakınsamış ve hata kareler ortalama değerleri giderek azaldığ görülmüştür.

Nelder Mead Simplex (NM) optimizasyon tekniği, diğer optimizasyon teknikleri ile karşılaştırıldığında, $\hat{\beta}_{1}$ bağımsız değişken katsayısının tahmin edilmesinde, örneklem büyüklükleri 100, 250 ve 500 iken hata kareler ortalama değerlerinin diğer yöntemlere göre daha yüksek olduğu gözlemlenmiştir.

Farklı örneklem büyüklüklerine ve parametre tahmin yöntemlerine göre optimizasyon tekniklerinin yakınsama oranları (\%) Tablo 3 'de gösterilmiştir.

Yakınsama oranlarına göre optimizasyon tekniklerinin performansları değerlendirildiğinde, Probit en büyük olabilirlik yöntemi, Tobit en büyük olabilirlik yöntemi ve Heckman iki aşamalı tahmin yönteminde \%100 yakınsama sağlayan optimizasyon tekniği Quasi Newton (QN) olduğu görülmektedir. 
Farklı Optimizasyon Teknikleri ve Örneklem Büyüklüklerinde Standart Tobit Modeli Tahmincilerinin Performansı üzerine Bir Simülasyon Çalışması

Tablo 1. Farkl optimizasyon teknikleri ve örneklem büyüklüklerine göre Probit en büyük olabilirlik, Tobit en büyük olabilirlik ve Heckman iki aşamalı tahmin yöntemlerinden elde edilen parametre tahminlerinin deneysel ortalamalarl

\begin{tabular}{|c|c|c|c|c|c|c|c|c|c|}
\hline $\begin{array}{l}\text { Tahmin } \\
\text { Yöntemleri }\end{array}$ & $\begin{array}{l}\text { Örneklem } \\
\text { Büyüklüğü }\end{array}$ & Parametre & NR & QN & CG & DD & NM & NRR & TR \\
\hline \multirow{8}{*}{ Probit } & \multirow{2}{*}{50} & $\widehat{\beta}_{0}$ & 1.1207 & 1.1167 & 1.0884 & 1.0980 & 1.1207 & 1.1207 & 1.1207 \\
\hline & & $\hat{\beta}_{1}$ & 1.1846 & 1.1607 & 1.1561 & 1.1227 & 1.1846 & 1.1846 & 1.1846 \\
\hline & \multirow{2}{*}{100} & $\widehat{\beta}_{0}$ & 1.0354 & 1.0342 & 1.0436 & 1.0354 & 1.0354 & 1.0354 & 1.0354 \\
\hline & & $\hat{\beta}_{1}$ & 1.0550 & 1.0566 & 1.0664 & 1.0550 & 1.0550 & 1.0550 & 1.0550 \\
\hline & \multirow{2}{*}{250} & $\widehat{\beta}_{0}$ & 1.0159 & 1.0105 & 1.0166 & 1.0159 & 1.0159 & 1.0159 & 1.0159 \\
\hline & & $\widehat{\beta}_{1}$ & 1.0192 & 1.0174 & 1.0271 & 1.0192 & 1.0192 & 1.0192 & 1.0192 \\
\hline & \multirow{2}{*}{500} & $\widehat{\beta}_{0}$ & 1.0078 & 1.0045 & 1.0131 & 1.0078 & 1.0078 & 1.0078 & 1.0078 \\
\hline & & $\widehat{\beta}_{1}$ & 1.0105 & 1.0032 & 1.0122 & 1.0105 & 1.0105 & 1.0105 & 1.0105 \\
\hline \multirow{12}{*}{ Tobit } & \multirow{3}{*}{50} & $\widehat{\beta}_{0}$ & 0.9916 & 0.9938 & 0.9849 & 0.9998 & 0.9916 & 0.9916 & 0.9916 \\
\hline & & $\hat{\beta}_{1}$ & 1.0189 & 1.0084 & 1.0178 & 1.0053 & 1.0189 & 1.0189 & 1.0189 \\
\hline & & $\hat{\sigma}$ & 0.9709 & 0.9787 & 0.9789 & 0.9799 & 0.9709 & 0.9709 & 0.9709 \\
\hline & \multirow{3}{*}{100} & $\widehat{\beta}_{0}$ & 0.9932 & 0.9936 & 0.9993 & 0.9938 & 0.9938 & 0.9938 & 0.9938 \\
\hline & & $\hat{\beta}_{1}$ & 1.0070 & 1.0064 & 1.0085 & 1.0059 & 1.0059 & 1.0059 & 1.0059 \\
\hline & & $\hat{\sigma}$ & 0.9898 & 0.9924 & 0.9870 & 0.9893 & 0.9893 & 0.9893 & 0.9893 \\
\hline & \multirow{3}{*}{250} & $\widehat{\beta}_{0}$ & 0.9990 & 0.9987 & 0.9979 & 0.9990 & 0.9994 & 0.9994 & 0.9994 \\
\hline & & $\widehat{\beta}_{1}$ & 1.0026 & 1.0005 & 1.0034 & 1.0018 & 1.0018 & 1.0018 & 1.0018 \\
\hline & & $\hat{\sigma}$ & 0.9971 & 0.9953 & 0.9937 & 0.9968 & 0.9967 & 0.9967 & 0.9967 \\
\hline & \multirow{3}{*}{500} & $\widehat{\beta}_{0}$ & 0.9982 & 0.9990 & 1.0020 & 0.9988 & 0.9985 & 0.9985 & 0.9985 \\
\hline & & $\hat{\beta}_{1}$ & 1.0005 & 0.9997 & 1.0009 & 1.0002 & 1.0004 & 1.0004 & 1.0004 \\
\hline & & $\hat{\sigma}$ & 0.9993 & 0.9988 & 0.9980 & 0.9992 & 0.9992 & 0.9992 & 0.9992 \\
\hline \multirow{12}{*}{ Heckman } & \multirow{3}{*}{50} & $\widehat{\beta}_{0}$ & 1.1550 & 1.1681 & 1.1457 & 1.1579 & 1.2364 & 1.1622 & 1.1505 \\
\hline & & $\widehat{\beta}_{1}$ & 0.8806 & 0.8591 & 0.8791 & 0.8697 & 0.8145 & 0.8735 & 0.8846 \\
\hline & & $\hat{\sigma}$ & 0.9169 & 0.9235 & 0.9230 & 0.9232 & 0.9058 & 0.9110 & 0.9161 \\
\hline & \multirow{3}{*}{100} & $\widehat{\beta}_{0}$ & 1.0834 & 1.0778 & 1.0842 & 1.0779 & 1.1774 & 1.1214 & 1.0968 \\
\hline & & $\hat{\beta}_{1}$ & 0.9321 & 0.9357 & 0.9356 & 0.9363 & 0.8576 & 0.8995 & 0.9235 \\
\hline & & $\hat{\sigma}$ & 0.9582 & 0.9605 & 0.9552 & 0.9578 & 0.9446 & 0.9530 & 0.9584 \\
\hline & \multirow{3}{*}{250} & $\widehat{\beta}_{0}$ & 1.0195 & 1.0195 & 1.0204 & 1.0179 & 1.1016 & 1.0225 & 1.0259 \\
\hline & & $\hat{\beta}_{1}$ & 0.9852 & 0.9826 & 0.9839 & 0.9866 & 0.9166 & 0.9809 & 0.9751 \\
\hline & & $\hat{\sigma}$ & 0.9865 & 0.9846 & 0.9827 & 0.9868 & 0.9687 & 0.9870 & 0.9888 \\
\hline & \multirow{3}{*}{500} & $\widehat{\beta}_{0}$ & 1.0029 & 1.0042 & 1.0051 & 1.0030 & 1.0335 & 1.0027 & 1.0047 \\
\hline & & $\hat{\beta}_{1}$ & 0.9969 & 0.9952 & 0.9981 & 0.9966 & 0.9687 & 0.9958 & 0.9958 \\
\hline & & $\hat{\sigma}$ & 0.9953 & 0.9944 & 0.9945 & 0.9948 & 0.9857 & 0.9954 & 0.9958 \\
\hline
\end{tabular}

NR: Newton Raphson; QN: Quasi Newton; CG: Conjugate Gradient; DD: Double Dogleg; NM: Nelder Mead Simplex; NRR: Newton Raphson Ridging (NRR); TR: Trust Region. 
Tablo 2. Farkl optimizasyon teknikleri ve örneklem büyüklüklerine göre Probit en büyük olabilirlik, Tobit en büyük olabilirlik ve Heckman iki aşamall tahmin yöntemlerinden elde edilen parametre tahminlerinin hata kareler ortalamalarl

\begin{tabular}{|c|c|c|c|c|c|c|c|c|c|}
\hline $\begin{array}{l}\text { Tahmin } \\
\text { Yöntemleri }\end{array}$ & $\begin{array}{l}\text { Örneklem } \\
\text { Büyüklüğü }\end{array}$ & Parametre & NR & QN & CG & DD & NM & NRR & TR \\
\hline \multirow{8}{*}{ Probit } & \multirow{2}{*}{50} & $\widehat{\beta}_{0}$ & 0.3818 & 0.2391 & 0.1277 & 0.1631 & 0.3817 & 0.3818 & 0.3818 \\
\hline & & $\hat{\beta}_{1}$ & 0.7288 & 0.2985 & 0.2110 & 0.2125 & 0.7285 & 0.7288 & 0.7287 \\
\hline & \multirow{2}{*}{100} & $\widehat{\beta}_{0}$ & 0.0443 & 0.0489 & 0.0425 & 0.0443 & 0.0443 & 0.0443 & 0.0443 \\
\hline & & $\hat{\beta}_{1}$ & 0.0585 & 0.0679 & 0.0654 & 0.0585 & 0.0585 & 0.0585 & 0.0585 \\
\hline & \multirow{2}{*}{250} & $\widehat{\beta}_{0}$ & 0.0152 & 0.0162 & 0.0146 & 0.0152 & 0.0152 & 0.0152 & 0.0152 \\
\hline & & $\hat{\beta}_{1}$ & 0.0214 & 0.0215 & 0.0224 & 0.0214 & 0.0214 & 0.0214 & 0.0214 \\
\hline & \multirow{2}{*}{500} & $\widehat{\beta}_{0}$ & 0.0074 & 0.0069 & 0.0073 & 0.0074 & 0.0074 & 0.0074 & 0.0074 \\
\hline & & $\widehat{\beta}_{1}$ & 0.0104 & 0.0098 & 0.0099 & 0.0104 & 0.0104 & 0.0104 & 0.0104 \\
\hline \multirow{12}{*}{ Tobit } & \multirow{3}{*}{50} & $\widehat{\beta}_{0}$ & 0.0240 & 0.0243 & 0.0239 & 0.0244 & 0.0240 & 0.0240 & 0.0240 \\
\hline & & $\hat{\beta}_{1}$ & 0.0289 & 0.0275 & 0.0284 & 0.0278 & 0.0289 & 0.0289 & 0.0289 \\
\hline & & $\hat{\sigma}$ & 0.0152 & 0.0146 & 0.0149 & 0.0153 & 0.0152 & 0.0152 & 0.0152 \\
\hline & \multirow{3}{*}{100} & $\widehat{\beta}_{0}$ & 0.0118 & 0.0124 & 0.0120 & 0.0117 & 0.0117 & 0.0117 & 0.0117 \\
\hline & & $\hat{\beta}_{1}$ & 0.0139 & 0.0130 & 0.0137 & 0.0140 & 0.0140 & 0.0140 & 0.0140 \\
\hline & & $\hat{\sigma}$ & 0.0073 & 0.0075 & 0.0067 & 0.0073 & 0.0073 & 0.0073 & 0.0073 \\
\hline & \multirow{3}{*}{250} & $\widehat{\beta}_{0}$ & 0.0044 & 0.0045 & 0.0048 & 0.0043 & 0.0044 & 0.0044 & 0.0044 \\
\hline & & $\hat{\beta}_{1}$ & 0.0056 & 0.0050 & 0.0052 & 0.0056 & 0.0056 & 0.0056 & 0.0056 \\
\hline & & $\hat{\sigma}$ & 0.0028 & 0.0029 & 0.0029 & 0.0028 & 0.0028 & 0.0028 & 0.0028 \\
\hline & \multirow{3}{*}{500} & $\widehat{\beta}_{0}$ & 0.0023 & 0.0023 & 0.0023 & 0.0023 & 0.0024 & 0.0024 & 0.0023 \\
\hline & & $\hat{\beta}_{1}$ & 0.0027 & 0.0027 & 0.0028 & 0.0027 & 0.0027 & 0.0027 & 0.0027 \\
\hline & & $\hat{\sigma}$ & 0.0013 & 0.0014 & 0.0014 & 0.0013 & 0.0013 & 0.0013 & 0.0013 \\
\hline \multirow{12}{*}{ Heckman } & \multirow{3}{*}{50} & $\hat{\beta}_{0}$ & 0.0984 & 0.1087 & 0.0991 & 0.1014 & 0.1545 & 0.1037 & 0.0954 \\
\hline & & $\widehat{\beta}_{1}$ & 0.0842 & 0.0965 & 0.0877 & 0.0846 & 0.1199 & 0.0889 & 0.0821 \\
\hline & & $\hat{\sigma}$ & 0.0213 & 0.0207 & 0.0200 & 0.0210 & 0.0232 & 0.0222 & 0.0214 \\
\hline & \multirow{3}{*}{100} & $\widehat{\beta}_{0}$ & 0.0551 & 0.0514 & 0.0533 & 0.0509 & 0.1137 & 0.0790 & 0.0640 \\
\hline & & $\hat{\beta}_{1}$ & 0.0437 & 0.0407 & 0.0419 & 0.0420 & 0.0833 & 0.0617 & 0.0504 \\
\hline & & $\hat{\sigma}$ & 0.0095 & 0.0097 & 0.0094 & 0.0095 & 0.0111 & 0.0108 & 0.0098 \\
\hline & \multirow{3}{*}{250} & $\widehat{\beta}_{0}$ & 0.0113 & 0.0124 & 0.0132 & 0.0106 & 0.0608 & 0.0131 & 0.0168 \\
\hline & & $\hat{\beta}_{1}$ & 0.0110 & 0.0108 & 0.0119 & 0.0110 & 0.0443 & 0.0129 & 0.0159 \\
\hline & & $\hat{\sigma}$ & 0.0031 & 0.0033 & 0.0035 & 0.0032 & 0.0051 & 0.0032 & 0.0033 \\
\hline & \multirow{3}{*}{500} & $\widehat{\beta}_{0}$ & 0.0030 & 0.0033 & 0.0023 & 0.0026 & 0.0205 & 0.0031 & 0.0037 \\
\hline & & $\widehat{\beta}_{1}$ & 0.0032 & 0.0035 & 0.0028 & 0.0030 & 0.0151 & 0.0033 & 0.0039 \\
\hline & & $\hat{\sigma}$ & 0.0013 & 0.0015 & 0.0015 & 0.0013 & 0.0024 & 0.0014 & 0.0014 \\
\hline
\end{tabular}

NR: Newton Raphson; QN: Quasi Newton; CG: Conjugate Gradient; DD: Double Dogleg; NM: Nelder Mead Simplex; NRR: Newton Raphson Ridging (NRR); TR: Trust Region 
Farklı Optimizasyon Teknikleri ve Örneklem Büyüklüklerinde Standart Tobit Modeli Tahmincilerinin Performansı üzerine Bir Simülasyon Çalışması

Tablo 3. Farkl örneklem büyüklüklerine ve parametre tahmin yöntemlerine göre optimizasyon tekniklerinin yakınsama oranları (\%)

\begin{tabular}{|c|c|c|c|c|c|c|c|c|}
\hline $\begin{array}{l}\text { Tahmin } \\
\text { Yöntemleri }\end{array}$ & $\begin{array}{l}\text { Örneklem } \\
\text { Büyüklüğü }\end{array}$ & NR & QN & CG & DD & NM & NRR & TR \\
\hline \multirow{4}{*}{ Probit } & 50 & 100 & 100 & 100 & 100 & 100 & 100 & 100 \\
\hline & 100 & 100 & 100 & 100 & 100 & 100 & 100 & 100 \\
\hline & 250 & 100 & 100 & 100 & 100 & 100 & 100 & 99.9 \\
\hline & 500 & 100 & 100 & 100 & 100 & 100 & 100 & 100 \\
\hline \multirow{4}{*}{ Tobit } & 50 & 100 & 100 & 100 & 100 & 100 & 100 & 100 \\
\hline & 100 & 98.9 & 100 & 100 & 100 & 100 & 100 & 100 \\
\hline & 250 & 98.8 & 100 & 100 & 98.4 & 100 & 100 & 100 \\
\hline & 500 & 99.5 & 100 & 100 & 94 & 100 & 100 & 99.9 \\
\hline \multirow{4}{*}{ Heckman } & 50 & 96.1 & 100 & 100 & 99.8 & 83.6 & 88.9 & 97.8 \\
\hline & 100 & 95.8 & 100 & 99.9 & 98.5 & 79.3 & 60.6 & 81.3 \\
\hline & 250 & 95.9 & 100 & 99.9 & 91.2 & 65.2 & 67.4 & 53.6 \\
\hline & 500 & 95.3 & 100 & 100 & 83.7 & 60.3 & 79.4 & 76.1 \\
\hline
\end{tabular}

NR: Newton Raphson; QN: Quasi Newton; CG: Conjugate Gradient; DD: Double Dogleg; NM: Nelder Mead Simplex; NRR: Newton Raphson Ridging (NRR); TR: Trust Region

Heckman iki aşamalı tahmin yönteminde, örneklem büyüklügü arttıkça Nelder Mead Simplex (NM) optimizasyon tekniğinin yakınsama oranları diğer yöntemlere göre giderek azaldığı görülmektedir. Sirası ile Trust Region (TR) ve Newton Raphson Ridging (NRR) teknikleri de diğer yöntemlere göre daha düşük yakınsama oranına sahiptir.

\section{Tartışma ve Sonuç}

Uluslararası alanlarda yapılan çalışmalarda Tobit modelleri uygulamalarının son yıllarda giderek arttığı gözlemlenmiştir. Ancak ülkemizde sağlık alanında yapılan araştırmalarda Tobit modellerinin geniş bir kullanım alanı bulunmamaktadır. Sağlık alanında yapılan araştırmalarda tahmin edilecek bağımlı değişken değerlerinin alttan ve üstten sansürlenmesi durumları ile çok sık karşılaşılmaktadır. $\mathrm{Bu}$ yapıyı göz önüne almadan farklı yöntemler ile istatistiksel analizler gerçekleştirildiğinde yanlış bulgular ve sonuçlar ortaya çıkmaktadır. Tobit modelinin sağlık alanında kullanımını artırarak doğru sonuçlar elde edilebilmesi mümkündür.

$\mathrm{Bu}$ çalışmada, standart tobit modelinin teorik yapıs1 ve parametre tahmin yöntemleri incelenmiş ve hangi yöntemin parametreleri tahmin etmede en iyi olduğunu belirlemek için simülasyon çalışması yapılmıştır.

Amemiya (1973), tobit modelinin logolabilirlik fonksiyonunun orijinal parametrelere göre yakınsama sağlayamadığını göstermiştir.
Amemiya'nın yaptığı çalışmayı tekrar inceleyen Olsen (1978) yeniden parametre dönüştürme işleminden sonra, tobit modelin, standart iteratif optimizasyon teknikleri ile logaritmik olabilirlik fonksiyonunun global maksimum değerine yakınsadığını göstermiştir (14, 15). Greene (1981), En küçük kareler yönteminin tobit modelin parametre tahmininde asimptotik yanlılı̆ga neden olacağını ve kullanılamayacağını ispatlamıştır (3). Schendler (2005), bağımlı değişkende sansür problemi ile karşılaşıldığında olabilirlik fonksiyonunun nasıl oluşturulacağını göstermiştir (10). Zhou (2007), tobit modelin semiparametrik ve parametrik olmayan tahminlerini incelemiştir (18). Kaaouachi ve El Melhaoui (2013), çalışmalarında tip 1 tobit modelinin parametrik tahmin edicileri olan en küçük kareler (LS) tahmincisi, Heckman (H) tahmincisi ve maksimum olabilirlik (ML) tahmincisini karşılaştırmıştır. $\mathrm{Bu}$ üç tahmincinin, rassal hata dağılımlarının bilinmesi durumunda tutarlı ve asimptotik olarak normal olduğunu ispatlamıştır. Ancak, bu özelliklerin hata dağılımının bilinmediği duruma karşı duyarlı olduğunu çeşitli simülasyon senaryoları ile belirlemiştir (19).

$\mathrm{Bu}$ çalışma sonucunda, örneklem büyüklüğü sabit tutulduğunda, Tobit en büyük olabilirlik yöntemi en küçük hata kareler ortalama değerlerine sahiptir ve Heckman iki aşamalı tahmin yöntemi bu yöntemi takip etmektedir. 
Örneklem büyüklüğü arttıkça başlangıç parametre değerlerine deneysel ortalamaların oldukça iyi yakınsadığı ve hata kareler ortalama değerlerinin giderek azaldığ 1 bilinmektedir.

Tobit en büyük olabilirlik yöntemi, örneklem büyüklüğü çok küçük olduğunda bile Probit en büyük olabilirlik yöntemi ve Heckman iki aşamalı tahmin yöntemine göre parametreleri tahmin etmede en iyi yöntemdir.

Optimizasyon tekniklerinin yakınsama oranları incelen-diğinde, en iyi performans gösteren optimizasyon tekniği Quasi Newton'dur (QN).
Heckman iki aşamalı tahmin yönteminde örneklem büyüklüğü arttıkça Nelder Mead Simplex (NM) optimizasyon tekniğinin yakınsama oranları giderek azalmıştır. $\mathrm{Bu}$ durum, parametre tahminlerinin hata kareler ortalama değerlerini artırmıştır. Nelder Mead Simplex (NM) optimizasyon tekniğini, sıras1 ile Trust Region (TR) ve Newton Raphson Ridging (NRR) teknikleri takip etmektedir. Heckman iki aşamalı tahmin yöntemi tercih edildiğinde bu optimizasyon tekniklerinin kullanılmaması önerilmektedir. 
Farklı Optimizasyon Teknikleri ve Örneklem Büyüklüklerinde Standart Tobit Modeli Tahmincilerinin Performansı üzerine Bir Simülasyon Çalışması

\section{KAYNAKLAR}

1. Tobin J. Estimation of relationships for limited dependent variables. Econometrica: journal of the Econometric Society. 1958:24-36, 0012-9682.

2. Goldberger AS. Econometric theory. Econometric theory. 1964.

3. Greene WH. On the asymptotic bias of the ordinary least squares estimator of the Tobit model. Econometrica: Journal of the Econometric Society. 1981:505-13, 00129682.

4. Powell JL. Least absolute deviations estimation for the censored regression model. Journal of Econometrics. 1984;25(3):303-25, 0304-4076.

5. Powell JL. Symmetrically trimmed least squares estimation for Tobit models. Econometrica: journal of the Econometric Society. 1986:1435-60, 0012-9682.

6. Tran BX, Ohinmaa A, Nguyen LT. Quality of life profile and psychometric properties of the EQ-5D-5L in HIV/AIDS patients. Health and quality of life outcomes. 2012;10(1):132.

7. Zhao Y, Li S-P, Liu L, Zhang J-L, Chen G. Does the choice of tariff matter?: A comparison of EQ-5D-5L utility scores using Chinese, UK, and Japanese tariffs on patients with psoriasis vulgaris in Central South China. Medicine. 2017;96(34).

8. Jin H, Wang B, Gao Q, Chao J, Wang S, Tian L, et al. Comparison between EQ-5D and SF-6D utility in rural residents of Jiangsu Province, China. PLoS One. 2012;7(7):e41550, 1932-6203.

9. Amemiya T. Tobit models: A survey. Journal of econometrics. 1984;24(1-2):3-61, 0304-4076.

10. Schnedler W. Likelihood estimation for censored random vectors. Econometric Reviews. 2005;24(2):195217, 0747-4938.
11. Huang T, Hughes E, editors. Nonlinear optimization in SAS/OR ${ }^{\circledR}$ software: Migrating from PROC NLP to PROC OPTMODEL2010.

12. Long SJ. SAGE Publications, Inc. Thousand Oaks, California. Regression models for categorical and limited dependent variables. 1997;7.

13. DeMaris A. Regression with social data: Modeling continuous and limited response variables: John Wiley \& Sons; 2004

14. Amemiya T. Regression analysis when the dependent variable is truncated normal. Econometrica: Journal of the Econometric Society. 1973:997-1016, 0012-9682.

15. Olsen RJ. Note on the uniqueness of the maximum likelihood estimator for the Tobit model. Econometrica: Journal of the Econometric Society. 1978:1211-5, 00129682.

16. Greene W. The behaviour of the maximum likelihood estimator of limited dependent variable models in the presence of fixed effects. The Econometrics Journal. 2004;7(1):98-119, 1368-423X.

17. Heckman J. The Common Structure of Statistical Models of Truncation, Sample Selection and Limited Dependent Variables and a Simple Estimator for Such Models. National Bureau of Economic Research, Inc; 1976.

18. Zhou X. Semiparametric and nonparametric estimation of Tobit models: Hong Kong University of Science and Technology (Hong Kong); 2007.

19. Kaaouachi A, El Melhaoui S. A Comparison of the Performance of Various Estimators of Parametric Type1 Tobit Model. Open Journal of Statistics. 2013;3(01):1. 